\title{
КОМПЛЕКСООБРАЗОВАНИЕ ВАНАДИЯ (IV) С ВАНАДИЕМ (V) В СИЛЬНОКИСЛОЙ СРЕДЕ
}

\author{
(Представил О. Эйзен)
}

В литературе описаны соединения ванадия со степенью окисления, промежуточной между ванадием (IV) и ванадием (V), - ванадил-ванадаты, образующиеся в слабокнслых $\left[{ }^{1-6}\right]$ и щелочных $\left[{ }^{7,8}\right]$ растворах. До сих пор подобных соединений в сильнокислой среде не было обнаружено. Данная работа посвящена определению состава, устойчивости и спектральных свойств ванадил-ванадата, образующегося в среде сильных кислот, концентрацией выше 0,5 моль/л.

\section{Экспериментальная часть}

В работе использовались: ванадат аммония ч.д.а., сульфат ванадила ч. д. а., вода бидистиллат. Концентрация ванадия (IV) и ванадия (V) в исходных растворах определялась титриметрически []. При определении исходной концентрации ванадия (IV) его предварительно окисляли до. ванадия (V) [10]. Для поддержания одинаковой ионной силы использовался хлорнокислый натрий, синтезированный из углекислого натрия и хлорной кислоты. Общая концентрация ванадия в изомолярных сериях была равной 0,1 моль/л. При расчетах ионной силы растворов учитывались константы диссоциации бисульфата при различных концентрациях серной кислоты ['1]. Измерения проводились при температуре $22 \pm 2{ }^{\circ} \mathrm{C}$. Спектры поглощения снимались на спектрофотометрах СФ-10 и «Specord UV VIS». Расчеты проводились с помощью ЭЦВМ «Видеотон 1010Б» и «Минск-32».

\section{Результаты и обсуждение}

Предварительно нами было установлено, что поглощение света растворами ванадия (IV) и ванадия (V) в изучаемых условиях подчиняется закону Бугера-Ламберта-Бера, хотя коэффициенты молярного погашения для ванадия (V) зависят от концентрации серной кислоты. Нами было обнаружено, что оптическая плотность растворов, содержащих смесь ванадия (IV) и ванадия (V) в сернокислой или хлорнокислой среде, во всем диапазоне видимого спектра отклоняется от-аддитивности, причем максимум отклонения наблюдается при соотношении ванадия (IV) и ванадия (V) равном 1:1. Наибольший прирост оптической плотности наблюдается в области длин волн 504-508 нм (рис. 1). Спектры поглощения в этой области были использованы для 


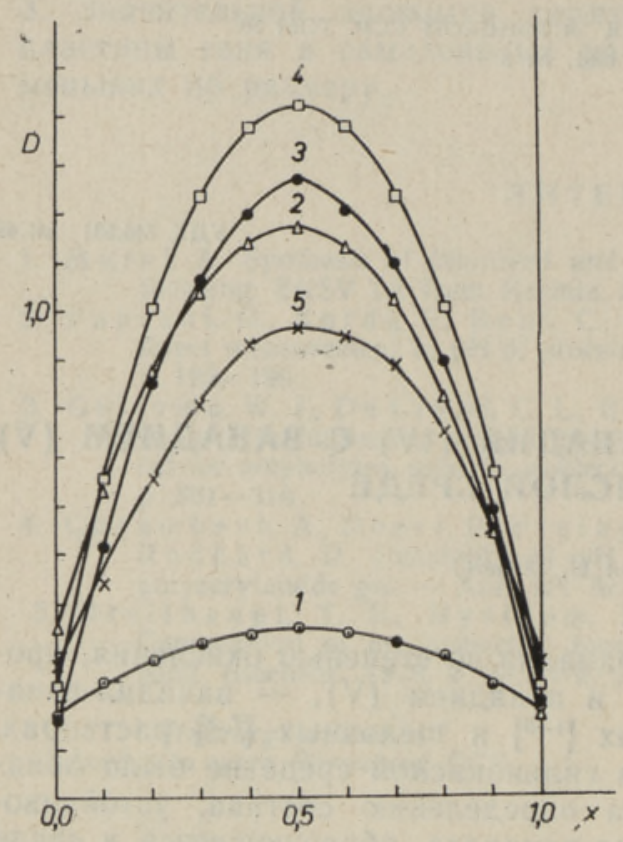

Рис. 1. Изменение оптической плотности в зависимости от состава изомолярной серии в системе: $C_{\mathrm{v}(\mathrm{IV})}+C_{\mathrm{v}(\mathrm{V})}=$ $=0,1$ моль $/ \Omega, \lambda=506 \mathrm{\mu M}, l=5,0 \mathrm{~cm}$.

$1-0,5$ моль/ $\Omega \mathrm{H}_{2} \mathrm{SO}_{4}, \mu=1,0 ; 2$. $1 \mu о л ь / \Omega \mathrm{H}_{2} \mathrm{SO}_{4}, \mu=5,8 ; 3-3 \mu о л ь / \Omega$ $\mathrm{H}_{2} \mathrm{SO}_{4}, \mu=5,8 ; 4-5$ моль/ $\mathrm{H}_{2} \mathrm{SO}_{4}$, $\mu=5,8 ; 5-5$ моль $/ \Omega \quad \mathrm{HClO}_{4}, \mu=5,8$. Фоновый электролит $\mathrm{NaClO}_{4}$.

расчета констант устойчивости $(K)$ ванадил-ванадатов и их молярных коэффициентов погашения $(\varepsilon)$. Полагая, что взаимодействие ванадия (IV) и ванадия (V) происходит по типу:

$$
A+B \rightleftarrows A B,
$$

можно записать выражение константы устойчивости образующегося комплекса:

$$
K=\frac{[A B]}{[A] \cdot[B]}=\frac{[A B]}{\left(C_{A}-[A B]\right)\left(C_{B}-[A B]\right)},
$$

где $C_{A}$ и $C_{B}$ - исходные концентрации реагентов; $[A],[B]$ и $[A B]-$ соответствующие равновесные концентрации.

Величина отклонения оптической плотности от аддитивности равна:

$$
D_{m}=D_{\text {экеп }}-C l\left[\varepsilon_{A} x+\varepsilon_{B}(1-x)\right] \text {, }
$$

где $C=C_{A}+C_{B}, x=\frac{C_{A}}{C}, 1-x=\frac{C_{B}}{C}$,

$\varepsilon_{A}, \varepsilon_{B}-$ коэффициенты молярного погашения соответственно компонента $A$ и $B$,

$l$ - толщина слоя.

Согласно $\left[{ }^{12}\right]$, для $i$-той точки изомолярной серии справедливо уравнение:

$$
D_{m i}=l \varepsilon_{m}[A B]=l \varepsilon_{m}\left(\frac{C+1 / k}{2}-\sqrt{\left(\frac{C+1 / k}{2}\right)^{2}-C^{2}\left(1-x_{i}\right) x_{i}},\right.
$$

где $\varepsilon_{m}=\varepsilon_{k}-\varepsilon_{A}-\varepsilon_{B}-$ прирост коэффициента молярного погашения;

$\varepsilon_{k}$ - коэффициент молярного погашения комплекса $[A B]$. 
Значения констант устойчивости ванадил-ванадата в сильнокислой среде $\left(C_{\mathrm{v}(\mathrm{Iv})}+C_{\mathrm{v}(\mathrm{v})}=0,1\right.$ моль $\left./ \Omega, \mathrm{t} 22+2{ }^{\circ} \mathrm{C}\right)$

\begin{tabular}{|c|c|c|c|c|c|c|}
\hline \multirow[b]{2}{*}{ № } & \multirow[b]{2}{*}{$\begin{array}{c}\text { Среда (фоновый } \\
\text { электролит } \mathrm{NaClO}_{4} \text { ) }\end{array}$} & \multirow[b]{2}{*}{$\mu$} & \multicolumn{2}{|c|}{ I метод } & \multicolumn{2}{|c|}{ II метод } \\
\hline & & & $\underset{M O A b / \Omega}{K}$ & $\lambda=506 \mathrm{m \mu}$ & $\begin{array}{c}K \\
M O \lambda b / \Omega\end{array}$ & $\lambda=\begin{array}{l}\Delta S^{2} \\
\lambda 06 \mathrm{Hm}\end{array}$ \\
\hline 1 & $0,5 \mu о л ь / \Omega \quad \mathrm{H}_{2} \mathrm{SO}_{4}$ & 1,0 & $0,5 \pm 0.2$ & 0,00003 & $1,0 \pm 0,4$ & 0,00002 \\
\hline 2 & 1,0 моль $/ \Omega \quad \mathrm{H}_{2} \mathrm{SO}_{4}$ & 5,8 & $1,1 \pm 0,8$ & 0,00038 & $0,7 \pm 0,4$ & 0,00035 \\
\hline 3 & $3,0 \mu о л ь / \Omega \mathrm{H}_{2} \mathrm{SO}_{4}$ & 5.8 & $1,2 \pm 0,7$ & 0,00020 & $1,1 \pm 0,1$ & 0,00024 \\
\hline 4 & $5,0 \wedge о л b / \Omega \quad \mathrm{H}_{2} \mathrm{SO}_{4}$ & 5,8 & $1,2 \pm 0,7$ & 0,00057 & $1,1 \pm 0,3$ & 0,00012 \\
\hline 5 & $5,0 \mathrm{MO} \mathrm{\Omega b} / \Omega \mathrm{H}_{2} \mathrm{SO}_{4}$ & 5,8 & $0,8 \pm 0,4$ & 0,00043 & $0,8 \pm 0,1$ & 0,00042 \\
\hline
\end{tabular}

Расчет $K$ и $\varepsilon_{k}$ проводился двумя методами. Первый метод описан в работе $\left[{ }^{13}\right]$ и заключается в минимизации дисперсии:

$$
\Delta S^{2}=\frac{1}{n} \sum_{i=1}^{n}\left(D_{m i}-D_{m i}^{\prime}\right)^{2}
$$

где $D_{m i}^{\prime}-$ прирост оптической плотности, полученный из экспериментальных данных;

$D_{m i}$ - прирост оптической плотности, вычисленный по уравнению (3);

$n$ - число экспериментальных точек.

При расчете по первому методу получены небольшие интервалы значений $K$ и $\varepsilon_{k}$ ванадил-ванадатов, при которых дисперсия минимальна и примерно одинакова, что свидетельствует о неоднозначности вычислений в указанных интервалах (таблица). Второй метод расчета основан на использовании уравнения:

$$
\frac{1}{\varepsilon_{m}^{2} l^{2}} D_{m i}^{2}-\frac{C+1 / K}{\varepsilon_{m} l} D_{m i}+C^{2} x_{i}\left(1-x_{i}\right)=0
$$

полученного из (1) и (3).

Приняв $C^{2} x_{i}\left(1-x_{i}\right)=y_{i}$, можем записать:

$$
y_{i}=-\frac{1}{\varepsilon_{m}^{2} l^{2}} D_{m i}^{2}+\frac{C+1 / K}{\varepsilon_{m} l} D_{m i}=a D_{m i}^{2}+b D_{m i},
$$

где $a=-\frac{1}{\varepsilon_{m}^{2} l^{2}} \quad$ и $\quad b=\frac{C+1 / K}{\varepsilon_{m} l}$.

Далее задача сводится к нахождению коэффициентов $a$ и $b$ полинома второй степени (6), у которого свободный член равен нулю. Для этого используются формулы, выведенные на основе метода наименьших квадратов (MНК) [14]. Расчет показал, что в нашем случае при максимальных значениях $D_{m i}$ квадратичный член полинома составляет не более $5 \%$ члена первой степени. Если пренебречь квадратичным членом, то полином (6) примет вид:

$$
y_{i}=b D_{m i} .
$$

Коэффициент $b$ можно определить графически или по МНК из условия: 


$$
\frac{\partial}{\partial D_{m i}} \sum_{i=1}^{n}\left(b D_{m i}-y_{i}\right)^{2}=0 .
$$

Значение $b$ вычисляется однократно для каждой серии экспериментальных точек. Из формул (1) и (3) следует:

$$
D_{m i}=l \varepsilon_{m}[A B]=l \varepsilon_{m} K[A][B] .
$$

Значение $\varepsilon_{m} K$ можно определить по МНК из условия:

$$
\begin{gathered}
\frac{\partial}{\partial \varepsilon_{m} K} \sum_{i=1}^{n}\left(D_{m i}-[A][B]\right)^{2}=0, \\
\varepsilon_{m} K=\frac{\sum_{i=1}^{n} D_{m i}[A][B]}{i \sum_{i=1}^{n}[A]^{2} \cdot[B]^{2}}
\end{gathered}
$$

Зная $\varepsilon_{m} K$ и величину $b=\frac{C+1 / K}{\varepsilon_{m} l}, \quad$ можно вычислить $\varepsilon_{m}$ и $K$. Поскольку значения $[A]$ и $[B]$, необходимые для расчета $\varepsilon_{m} K$, неизвестны, то в первом приближении принималось $[A]=C_{A}$ и $[B]=C_{B}$. Полученное значение $\varepsilon_{m} K$ использовалось для уточнения значений $[A]$ и $[B]$, после чего весь цикл повторялся до тех пор, пока не было соблюдено условие: $\varepsilon_{m}-\varepsilon_{m}^{\prime} \leqslant 0,001$, где $\varepsilon_{m}^{\prime}-$ значение коэффициента молярного погашения предыдущего шага итерации. При этом значения констант устойчивости, вычисленных в последнем цикле, отличались от полученных в предыдущем цикле не более чем на $1 \%$. Константы устойчивости, полученные по второму методу, попадают в интервал значений $K$, полученных по первому методу (см. таблицу). Дальнейшая обработка результатов производилась на основе констант, рассчитанных по второму методу, поскольку он обеспечивал однозначность решения.

Используя эти значения констант, по уравнению (1) мы вычислили равновесные концентрации ванадия (IV) и ванадия (V). Прямолинейный характер зависимости $D_{m i}$ от произведения вычисленных равновесных концентраций ванадия (IV) и ванадия (V) подтверждает образование в изучаемой системе комплексных частиц с соотношением ванадия (IV) и ванадия (V) равным $1: 1$, а следовательно, и пригодность уравнения (3) для расчетов. Используя это уравнение и полученные константы устойчивости, исходя из спектров поглощения смесей ванадия (IV) и ванадия (V), мы рассчитали спектры поглощения ванадил-ванадатов (рис. 2). Они отличаются от спектров исходных растворов ванадия (IV) и ванадия (V) наличием максимумов при 570 и $670 \mathrm{\mu м.}$.

Следует отметить, что полученные нами константы устойчивости ванадил-ванадатного комплекса условны и справедливы только для указанной ионной среды, так как выражение константы, использованное в расчете, включает общие равновесные концентрации ванадия (IV) и ванадия (V) безотносительно к каким-то определенным их формам. Истинные константы устойчивости, в принципе, могут быть рассчитаны из этих условных констант при известном составе исходных частиц реагентов и полном составе ванадил-ванадатного комплекса. Имеющиеся данные о состоянии ванадия (IV) и ванадия (V) в условиях, близких к изученным, дают возможность пока делать предположения о вероятной формуле обнаруженного нами ванадил- 


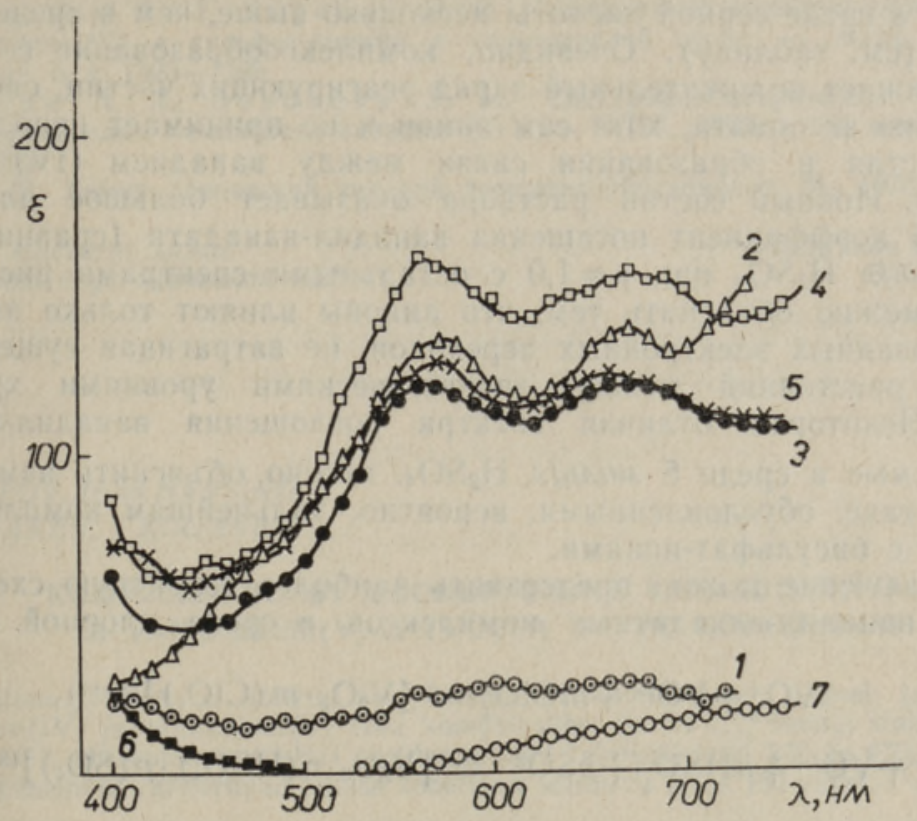

Рис. 2. Спектры поглощения ванадил-ванадатов при различной кислотности. Номера кривых от 1 до 5 соответствуют номерам серий в таблице; 6 - раствор $\mathrm{NH}_{4} \mathrm{VO}_{3}$ и 7 - раствор $\mathrm{VOSO}_{4}$ в 5 моль/ $\Omega \mathrm{H}_{2} \mathrm{SO}_{4}$ при $\mu=5,8$.

ванадата. Из литературы известно, что ванадий (V) в сернокислых растворах может образовывать сульфатные комплексы $\mathrm{VO}_{2} \mathrm{SO}_{4}^{-}$и $\mathrm{VO}_{2} \mathrm{H}\left(\mathrm{SO}_{4}\right)_{2}^{2-}\left[{ }^{15,16}\right]$. Установлено также, что ванадий (IV) в сернокислой среде образует комплексы $\mathrm{VOSO}_{4}, \mathrm{VO}\left(\mathrm{SO}_{4}\right)_{2}^{2-}$ и $\mathrm{VOHSO}_{4}^{-}\left[{ }^{17}\right]$. Часто причиной образования полиядерных соединений, включающих в себя ионы одного и того же металла в разных степенях окисления, оказываются мостиковые анионы $\left[{ }^{18}\right]$. Для образования ванадил-ванадатов в изученных нами условиях такими мостиковыми анионами могут быть $\mathrm{SO}_{4}^{2-}, \mathrm{HSO}_{4}^{-}$и $\mathrm{ClO}_{4}^{-}$. Однако следует заметить, что спектры поглощения ванадил-ванадатов в среде серной и хлорной кислот мало отличаются друг от друга. Следовательно, природа полос при 570 и 670 нм слабо зависит от указанных анионов. По-видимому, эти полосы обусловлены переходами между уровнями, которые включают в себя главным образом $d$-орбитали обоих атомов ванадия, связанных между собой кислородным мостиком

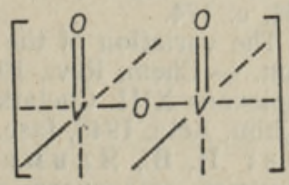

Интересно отметить, что, несмотря на более высокий коэффициент активности перхлорат-ионов по сравнению с коэффициентами активности сульфат- и бисульфат-ионов, константы устойчивости ванадил- 
ванадата в среде серной кислоты несколько выше, чем в среде хлорной кислоты (см. таблицу). Очевидно, комплексообразование с сульфатионом снижает положительный заряд реагирующих частиц, способствуя упрочнению ассоциата, хотя сам анион и не принимает непосредственного участия в образовании связи между ванадием (IV) и ванадием (V). Ионный состав раствора оказывает большое влияние на молярный коэффициент погашения ванадил-ванадата (сравним спектр в 0,5 моль/ $\Omega \mathrm{H}_{2} \mathrm{SO}_{4}$ при $\mu=1,0$ с остальными спектрами рис. 2). Это явление можно объяснить тем, что анионы влияют только на вероятность указанных электронных переходов, не затрагивая существенным образом расстояний между энергетическими уровнями хромофора $\mathrm{V}_{2} \mathrm{O}_{3}^{3+}$. Некоторые отличия спектра поглощения ванадил-ванадата, наблюдаемые в среде 5 моль/ $\mathrm{H}_{2} \mathrm{SO}_{4}$, можно объяснить изменениями в его составе, обусловленными, вероятно, дальнейшим комплексообразованием с бисульфат-ионами.

В заключение можно представить наиболее вероятную схему образования ванадил-ванадатных комплексов в среде хлорной и серной кислот:

$$
\begin{gathered}
\left.\mathrm{VO}_{2}^{+}+\mathrm{VO}^{2+}+m \mathrm{ClO}_{4}^{-} \rightleftarrows\left[\mathrm{V}_{2} \mathrm{O}_{3} \cdot m\left(\mathrm{ClO}_{4}\right)\right]^{(3-m}\right) \\
\mathrm{VO}_{2}^{+}+\mathrm{VO}^{2+}+n \mathrm{HSO}_{4}^{-}+p \mathrm{SO}_{4}^{2-} \rightleftarrows\left[\mathrm{V}_{2} \mathrm{O}_{3} \cdot n\left(\mathrm{HSO}_{4}\right) \cdot p\left(\mathrm{SO}_{4}\right)\right]^{(3-n-3 p)} .
\end{gathered}
$$

\section{Л И Т ЕР А Т У Р А}

1. Ostrowetsky, S., Souchay, M. P. Chimie minérale. Sur la constitution des isopolyvanadates mixtes entre $\mathrm{pH} 4,5$ et 6,5 . - Compt. Rend. Acad. Sci., 1963, t. $257, \mathrm{~N} 14$, p. $2036-2039$.

2. Ми ркин В. А., Козловский М. Т. Изучение состава и свойств ванадилванадатов методом спектрофотометрии. - Докл. АН СССР, 1963, т. 150 , № 2 , c. $317-320$.

3. S a rm a, P. L., D a vis, M. S. Some intermediates in the reaction between vanadium (IV) and vanadium (V). - Z . anorg. allgem. Chem., 1967, Bd. 353, S. 324-328.

4. Heitner-Wigruim, C., Selbin, J. A new mixed valence compound of vanadium. - J. Inorg. Nucl. Chem., 1963, v. 30, N 12, p. 3181-3188.

5. Ostrowets ky, S. Contribution à l'étude des isopolymolybdates et isopolyvanadates réduits. III. Isopolyvanadates réduits en milieu acide. - Bull. Soc. Chim. France, 1964, fasc. 5, p. $1018-1035$.

6. Крисс Е. Е., Я ци ми рский К. Б., Курбатова Г. Т. Спектры поглощения ванадил-ванадатов в области рН 2,5-6,0. - ЖПХ, 1976, т. 21, вып. 8, c. $2075-2080$.

7. Ostrow et sky, S. Contribution à l'étude des isopolymolybdates et isopolyvanadates réduits. II. Isopolyvanadates réduits en milieu alcalin. - Bull. Soc. Chim. France, 1964 , fasc. 5, p. $1012-1018$.

8. Ostrowetsky, S., Souchay, M. P. Chimie minérale. Sur la constitution des isopolyvanadates réduits en milieu alcalin. - Compt. Rend. Acad. Sci., 1963, t. $256, \mathrm{~N} 25$, p. $5355-5358$.

9. Сусленникова В. М., Киселева Е. К. Руководство по приготовлению титрованных растворов. Л., 1968 , с. $96-97$.

10. Ляликав Ю. С., Сакунов В. И., Ткаченко Н. С. Анализ железных и марганцевых руд. М., 1954 , с. 174.

11. Young, T. F., Blatz, L. A. The variation of the properties of electrolytic solutions with degrees of dissociation. - Chem. Rev., 1949, v. 44, N 1, p. 93-115.

12. S chwarzen b a ch, G. Komplexone XIII. Chelatkomplexe des Kobalts mit und ohne Fremdliganden. - Helv. Chim. Acta, 1949, fasc. 32, p. 839-853.

13. Фи липпов А. П., Х я р синг И. В., Яцим и рский К. Б. Расчет константы устойчивости и коэффициентов молярного погашения комплексных соединений типа $A B$ по данным изомолярных серий с применением ЭВМ. - Укр. хим. ж., 1974 , т. 40 , вып. 8 , с. $787-791$.

14. L e vi n, M., U I m, S. Arvutusmeetodite käsiraamat. Tln., 1966, lk. 85-87.

15. Ив в кин А. А. Комплексные ионы пятивалентного ванадия с хлор- и сульфатионами. - ЖПХ, 1966, т. 39, № 2, с. 277-284. 
16. Филиппов А. П., Х я рсин г И. В. Нсследование комплексообразования ванадия (V) с сульфат-ионами в сильнокислой среде. - ЖПХ, 1978, т. 23, вып. 6, с. $1523-1528$.

17. Ивакин А. А., В оронов а Э. М. Спектрофотометрическое исследование сернокислых комплексов ванадия (IV). - ЖПХ, 1973, т. 18, вып. 7, с. $1809-$ 1812.

18. Бск М. Химия равновесий реакций комплексообразования. М., 1973, с. $261-263$.

Ннститут химии

Академии наук Эстонской ССР
Поступнла в редакцию $8 / \mathrm{X} 1980$

A. FILIPPOV, Irina HARSING,

Ene KIRJANEN, I. KIRJANEN

\section{KOMPLEKSOHENDI MOODUSTUMINE VANAADIUM(IV)-st JA VANAADIUM(V)-st TUGEVA HAPPE KESKKONNAS}

Spektrofotomeetriliselt on uuritud kompleksühendi moodustumist väävel- ja kloorhappes. Vanaadium(IV) ja vanaadium(V) suhe kompleksühendis on 1:1, ühendi stabiilsuskonstant väike $(\mathrm{K} \approx 1)$, absorptsioonispektri neeldumisribad lainepikkustel 570 ja $670 \mathrm{~nm}$. Ekstinktsioonikoefitsiendid varieeruvad lahuse koostisest sõltuvalt 20 ja 160 vahel.

A. FILIPPOV, Irina HARSING,

Ene KIRJANEN, I. KIRJANEN

\section{COMPLEX FORMATION BETWEEN VANADIUM (IV) AND VANADIUM (V) IN HIGHLY ACID SOLUTION}

Complex formation between vanadium (IV) and vanadium (V) in sulphuric acid and hydrochloric acid solution with $\mu=5.8$ is studied spectrophotometrically. The ratio of $V($ IV): $: V(V)$ in the complex is $1: 1$. The stability constant and absorption spectrum of the complex are computed, the value of $\mathrm{K}$ being small $(\sim 1)$ and absorption spectrum having two bands at $\lambda_{\max } 570$ and $670 \mathrm{~m} \mu$. Extinction coefficients of these bands vary within 20 and 160 , being dependent on the ionic composition of the solution. 